\title{
La Educación Física escolar en la promoción de la actividad física orientada a la salud en la adolescencia: una revisión sistemática de programas de intervención
}

\section{The role of Physical Education in the promotion of health-oriented physical activity in adolescence: a systematic review of intervention programs}

\author{
María José CAMACHO-MIÑANO, Emilia FERNÁNDEZ GARCÍA, \\ Elena RAMÍREZ RICO y Julia BLÁNDEZ ÁNGEL \\ Universidad Complutense de Madrid
}

Recibido: Marzo 2012

Aceptado: Junio 2012

\section{Resumen}

Los elevados niveles de sedentarismo y obesidad en la edad adolescente han motivado que la promoción de la Actividad Física (AF) orientada hacia la salud se constituya como uno de los retos de la escuela actual y de la Educación Física (EF) en particular.

El presente trabajo realiza una revisión sistemática de programas de intervención que incorporan la EF como uno de sus componentes. Específicamente, este trabajo da respuesta a los siguientes interrogantes: ¿Qué papel desempeña la EF escolar en el conjunto de los programas de intervención?, ¿Cuáles son los componentes básicos que integran este área curricular en estos programas?, y ¿Cuál es el rol del profesorado de EF en su implementación y desarrollo? Se realizó una búsqueda sistemática de literatura en cinco bases de datos completada con una búsqueda manual de referencias, para identificar estudios de diseño experimental o cuasiexperimental, desarrollados en centros escolares de Educación Secundaria y publicados en revistas científicas hasta diciembre de 2011. Un total de 27 referencias correspondientes a 15 programas de intervención cumplieron los criterios de inclusión establecidos.

Los resultados avalan la conveniencia de que las intervenciones de promoción de la $\mathrm{AF}$ orientada hacia la salud en la edad adolescente incorporen la EF escolar como uno de sus elementos clave, revalorizándose así la contribución única y específica de esta asignatura en el 
currículum escolar. Dentro de este contexto, el profesorado de EF se revela como el agente principal para implementar este tipo de programas, adaptándolos a la realidad educativa de cada centro escolar y asegurando su continuidad.

Palabras clave: Educación Física, programas de intervención, promoción de actividad física, profesorado de Educación Física, estudiantes de Educación Secundaria.

\begin{abstract}
The high levels of sedentary behavior and obesity during adolescence have caused the promotion of health-oriented Physical Activity (PA) to constitute one of the challenges of current schooling and of Physical Education (PE) in particular.

A narrative systematic review was conducted to describe the available evidence of intervention programs that incorporate $\mathrm{PE}$ as one of their components. Specifically, this paper answers the following questions: What is the role of PE in school intervention programs as a whole? What are the basic components that make up this area of the curriculum in these programs? And what is the role of PE teachers in their implementation and development? A systematic literature search was conducted in five databases supplemented by a manual search of references in order to identify studies with experimental or quasi-experimental design, developed in Secondary Education schools and published in scientific journals until December 2011. A total of 27 references for 15 intervention programs met the inclusion criteria.

The results support the appropriateness of interventions to promote health-oriented PA during adolescence with $\mathrm{PE}$ as one of its key elements, appreciating the unique and specific contribution of this subject in the school curriculum. Within this context, the PE teacher is shown to be the main agent for implementing such programs, adapting them to each school center and ensuring their continuity.
\end{abstract}

Keywords: Physical Education, intervention programs, physical activity promotion, Physical Education Teacher, Secondary School students.

La participación regular en actividades físicas proporciona a la población adolescente múltiples beneficios para su salud, entre los que destacan una mejora de la condición física cardiovascular, una mejor salud ósea y un mayor bienestar psicológico, convirtiéndose en un aspecto clave para la prevención de la obesidad (Janssen \& LeBlanc, 2010; Strong et al., 2005). Para que estos beneficios se hagan efectivos se ha recomendado que los jóvenes realicen al menos 60 minutos de Actividad Física (AF) de intensidad moderada a vigorosa la mayoría de los días de la semana (WHO, 2010).

No obstante, son numerosos los estudios que han constatado que casi la mitad de los chicos y una proporción aún mayor de chicas no alcanzan los citados niveles de AF (Butcher et al., 2008; Currie et al., 2008; Pate et al., 2002; Riddoch \& Andersen, 2004). En España, se ha encontrado que 56,9\% de los chicos y 38,9\% de las chicas cumple con las recomendaciones de AF (Román et al., 2008). El estudio de Fernández et al. (2007) constató igualmente que un 42,1\% de los chicos y sólo un 18,4\% de las chicas de entre 10 y 16 años alcanzaba los niveles de AF necesarios para su salud, siendo la 
transición de la enseñanza primaria a la secundaria el momento en el que muchas de ellas abandonan la práctica.

La edad adolescente se revela como etapa crítica ya que, según se ha confirmado a través de estudios longitudinales, se produce un marcado descenso de la práctica de AF (Kahn et al., 2008; Kimm et al., 2000; Sallis, 2000), precisamente en unos años en los que se consolidan hábitos de práctica que tienden a perpetuarse en la edad adulta (Gordon-Larsen et al., 2004; Telama, 2009).

En consecuencia, el desarrollo de intervenciones para la promoción de la AF entre los jóvenes se ha convertido en una prioridad, señalándose la escuela como el entorno más apropiado para ello (Kahn et al., 2002). Además de que los jóvenes pasan gran parte de su jornada en el centro escolar, esta afirmación se basa en la existencia en las escuelas de los siguientes elementos clave: profesorado sensibilizado con la problemática de la salud de los escolares, estructuras organizativas e instalaciones apropiadas para promover la AF y la existencia de materias curriculares que incorporan esta orientación (Pate et al., 2006; Wechsler et al., 2000). Específicamente la Educación Física (EF) curricular se constituye como el componente fundamental para la promoción de la AF orientada hacia la salud en la edad adolescente (Slingerland, \& Borghouts, 2012; Ahrabi-Fard \& Matvienko, 2005; Wallhead \& Buckworth, 2004).

No obstante, existen una serie de obstáculos importantes para que pueda asumir adecuadamente esta función, como son la baja consideración que posee la EF en el estatus educativo, el papel marginal que ocupa el profesorado de esta asignatura, la escasez del valor educativo que se le reconoce al área y el poco tiempo que se le asigna dentro del horario escolar (Estrada, 2010; Hardman, 2003; McKenzie \& Lounsbery, 2009). Desde diversas instituciones se ha realizado un llamamiento para revalorizar la EF curricular, puesto que realiza una contribución única y específica al desarrollo y la salud integral del alumnado que no se aborda desde otras áreas curriculares (ICSSPE, 2005; NASPE, 2011; Parlamento Europeo, 2007). Además resulta un área clave para el cumplimiento de las recomendaciones de AF en la edad adolescente, tanto por el tiempo de AF que puede proporcionar (Fairclough \& Stratton, 2005; Pate et al., 2007) como por el hecho de que sus objetivos educativos se centran en la adquisición de los conocimientos y habilidades necesarias para la adopción de un estilo de vida activo (Ahrabi-Fard \& Matvienko, 2005).

Este inestimable papel de la EF escolar ha justificado su inclusión como un componente clave dentro de los programas de intervención para la promoción de la AF orienta hacia la salud. Este trabajo tiene como propósito realizar una revisión sistemática de los programas desarrollados en el marco escolar que integran la EF curricular como uno de sus componentes. En concreto, trata de darse respuesta a los siguientes interrogantes: ¿Qué papel desempeña el área de EF en el conjunto de los programas de intervención?, ¿Cuáles son los aspectos básicos que integran esta área curricular?, y ¿Cuál es el rol del profesorado de EF en la implementación y desarrollo de este tipo de programas? 


\section{Metodología}

La identificación de programas de intervención de EF orientada hacia la salud y dirigidos específicamente a la población adolescente se llevó a cabo a través de una revisión sistemática de la literatura científica mediante una búsqueda bibliográfica realizada en cinco bases de datos electrónicas: MedLine, PubMed, PsychInfo, Web of Science y SPORT-Discus. Se realizó una estrategia de búsqueda individualizada en las diferentes bases de datos utilizando una combinación de las siguientes palabras clave: "exercise, physical activity, sport, physical education, adolescents, intervention and program". La localización de documentación relevante se completó a través de una búsqueda manual de referencias, realizada tanto sobre los artículos de interés previamente localizados, como sobre los artículos de revisión centrados en la promoción de AF en la población adolescente (De Meester et al., 2009; Jago \& Baranowski, 2004; Lubans et al., 2008; Lubans et al., 2009; Timperio et al., 2004; Van Sluijs et al., 2008).

La selección de los programas de intervención que finalmente se incluyeron en este trabajo se realizó a través de la aplicación de una serie de criterios de inclusión, definidos en función de los propósitos de este trabajo y que habían de ser cumplidos en su totalidad por los estudios previamente identificados. Los criterios de inclusión fueron los siguientes:

- Intervenciones centradas en la población adolescente, entendiéndose por este colectivo aquellos chicos y chicas de edades comprendidas entre los 12 y los 18 años de edad.

- Dirigidas a sujetos sanos, es decir, que no sufren problemas médicos específicos (p.e., obesidad o diabetes).

- Con un componente de la intervención dirigido específicamente al incremento de los niveles de AF.

- Estudios desarrollados en los centros escolares de Educación Secundaria y que incluyan específicamente un componente implementado en la EF escolar como área curricular.

- Estudios de diseño experimental o cuasiexperimental.

- Publicados en revistas científicas desde 1990 hasta diciembre de 2011, excluyéndose resúmenes, comunicaciones de congresos, estudios pilotos o aquellos estudios de carácter cualitativo.

La información relevante de cada trabajo fue analizada usando una planilla estandarizada y consensuada por los autores de este trabajo que recogía información sobre los datos generales del estudio, la muestra, las características del diseño metodológico, los componentes de la intervención y los agentes implicados en su implementación. 


\section{Resultados}

Un total de 15 programas de intervención (27 referencias) cumplieron los criterios de inclusión establecidos. Una síntesis de estos estudios se muestra en la Tabla 1, que incluye información sobre la autoría, año, nombre del estudio, muestra, diseño, así como una breve descripción de la intervención.

\begin{tabular}{|c|c|c|c|}
\hline$\underset{\text { [ref. principal] }}{\text { Estudio }}$ & Muestra & Diseño del estudio & Descripción de la intervención \\
\hline $\begin{array}{c}\text { Neumark- } \\
\text { Sztainer (2010) } \\
\text { New Moves-2 } \\
\text { (Neumark-Sztainer et } \\
\text { al., 2010) }\end{array}$ & $\begin{array}{l}-256 \text { chicas } \\
\text { inactivas } \\
-15.8 \text { años } \\
(\mathrm{SD}=1.2) \\
\text { - USA }\end{array}$ & $\begin{array}{l}\text { - } 16 \text { semanas } \\
\text { - RCT } \\
\text { - I ( } 3 \text { centros, } 177 \\
\text { chicas }) \text {; }(3,174)\end{array}$ & $\begin{array}{l}\text { Clases de EF solo para chicas (4 días/semana) con } \\
\text { AF adecuadas a chicas de diferentes niveles de } \\
\text { competencia motriz y sesiones educativas con } \\
\text { debates sobre apoyo social/empoderamiento y } \\
\text { nutrición. Se envían cartas a los padres, bolsas con } \\
\text { material para casa y se crean vínculos con la } \\
\text { comunidad. Las chicas también reciben sesiones de } \\
\text { orientación individuales. }\end{array}$ \\
\hline $\begin{array}{c}\text { McMurray } \\
\text { (2009) } \\
\text { HEALTHY } \\
\text { study } \text { (McMurray et }_{\text {al., 2009b) }}\end{array}$ & $\begin{array}{l}\text { - } 531 \text { adolesc. } \\
\text { - Grado } 6 \\
\text { - USA }\end{array}$ & $\begin{array}{l}-2 \text { años } \\
\text { - RCT } \\
\text { - I (19centros, } 265 \\
\text { jóvenes); C (20, } \\
\text { 266) }\end{array}$ & $\begin{array}{l}\text { Consta de } 4 \text { componentes: nutrición, con la mejora } \\
\text { de la calidad de la alimentación en el centro } \\
\text { escolar; clases de EF con sesiones de formación } \\
\text { para que el profesorado imparta las sesiones } \\
\text { establecidas, personal ayudante y provisión de } \\
\text { material; comportamental, a través de actividades } \\
\text { educativas, cartas a los padres y bolsas con } \\
\text { material; y componente de comunicación a través } \\
\text { de mensajes y eventos y con estudiantes como } \\
\text { líderes. }\end{array}$ \\
\hline $\begin{array}{l}\text { Jones (2008) } \\
\text { IMPACT (Jones et } \\
\text { al., 2008) }\end{array}$ & $\begin{array}{l}\text { - } 718 \text { chicas } \\
-11.6 \text { años } \\
(\mathrm{SD}=0.4) \\
\text { - USA }\end{array}$ & $\begin{array}{l}\text { - } 18 \text { meses } \\
\text { - RCT. } \\
\text { - I ( } 6 \text { escuelas, } 371 \\
\text { chicas); C }(6,347)\end{array}$ & $\begin{array}{l}\text { Dentro de la clase habitual de EF se realizan en el } \\
\text { calentamiento } 10 \text { minutos de actividades de alto } \\
\text { impacto. Un currículum de Educación para la Salud } \\
\text { se orienta a promover este tipo de actividades y el } \\
\text { consumo de alimentos ricos en calcio. }\end{array}$ \\
\hline $\begin{array}{c}\text { Taymoori } \\
\underset{(2008)}{\text { al., 2008) }}\end{array}$ & $\begin{array}{l}\text { - } 161 \text { chicas } \\
\text { sedentarias } \\
-14.8 \text { años } \\
(\mathrm{SD}=0.4) \\
\text { - Irán }\end{array}$ & $\begin{array}{l}\text { - } 6 \text { meses. } \\
\text { - RCT } \\
\text { - Grupo I1 (1 } \\
\text { escuela, } 55 \text { chicas }) \text {, } \\
\text { Grupo I2 }(1,54), \text { C } \\
(1,52)\end{array}$ & $\begin{array}{l}\text { I basada en el MPS: en EF, sesiones educativas en } \\
\text { grupo y con cada chica en particular, para ayudarles } \\
\text { a establecer metas sobre AF. Sesiones educativas } \\
\text { con profesorado y madres, y una salida al medio } \\
\text { natural, completan el programa. } \\
\text { I basada en el TTM: Igual, más dos sesiones } \\
\text { educativas sobre control de estímulos y } \\
\text { condicionamiento. }\end{array}$ \\
\hline $\begin{array}{l}\text { Webber (2008) } \\
\text { TAAG } \\
\text { al., 2008) }\end{array}$ & $\begin{array}{l}3 \text { Muestras } \\
\text { transversales de } \\
\text { chicas }(\mathrm{n}=1721 \\
\text { hasta } \mathrm{n}=3504) \\
\text { - Grados 6-8 (12- } \\
\text { 14 años) } \\
\text { - USA }\end{array}$ & $\begin{array}{l}-2 \text { años }+1 \text { de } \\
\text { mantenimiento } \\
\text { - RCT } \\
\text { - I ( } 18 \text { schools }), C \\
(18)\end{array}$ & $\begin{array}{l}\text { Según el modelo socioecológico, se basa en la } \\
\text { creación de un equipo de profesores en el centro } \\
\text { escolar que, liderados por el profesor de EF, } \\
\text { desarrollan la intervención. Esta incorpora } \\
\text { actuaciones en la clase de EF, un currículum de } \\
\text { Educación para la salud y el desarrollo y promoción } \\
\text { de programas para chicas, reforzando los vínculos } \\
\text { entre la escuela y la comunidad. }\end{array}$ \\
\hline
\end{tabular}




\begin{tabular}{|c|c|c|c|}
\hline $\begin{array}{l}\text { Singh (2006) } \\
\text { Doit (Singh et al., } \\
\text { 2006) }\end{array}$ & $\begin{array}{l}\text { - } 978 \text { adolesc. } \\
-\approx 12.7 \text { años } \\
\text { - Holanda }\end{array}$ & $\begin{array}{l}-8 \text { meses } \\
\text { - RCT } \\
\text { - I ( } 10 \text { escuelas, } 600 \\
\text { estudiantes); C ( } 8 \text {, } \\
453)\end{array}$ & $\begin{array}{l}\text { Incorpora un programa educativo integrado en las } \\
\text { asignaturas de EF y de Biología, junto con un } \\
\text { programa individualizado por ordenador que } \\
\text { orienta a los jóvenes en su proceso de cambio según } \\
\text { el TTM. Se financia a las escuelas para que } \\
\text { ofrezcan horas adicionales de práctica de AF. }\end{array}$ \\
\hline $\begin{array}{l}\text { Young (2006) } \\
\text { Life Skills } \\
\text { Interven. (Young et } \\
\text { al., 2006) }\end{array}$ & $\begin{array}{l}-221 \text { chicas } \\
-13.8 \text { años } \\
(\mathrm{SD}=0.5) \\
\text { - USA }\end{array}$ & $\begin{array}{l}\text { - } 8 \text { meses } \\
\text { - RCT } \\
\text { - I (116); C (105) }\end{array}$ & $\begin{array}{l}\text { En la clase de EF se desarrollan actividades físicas } \\
\text { innovadoras, no competitivas y orientadas a la } \\
\text { adquisición de habilidades motrices y al incremento } \\
\text { de AF fuera y dentro de las clases; un currículum } \\
\text { educativo refuerza este enfoque. }\end{array}$ \\
\hline $\begin{array}{c}\text { Tsoorbatzoudis } \\
\text { (2005) } \\
\text { (Tsorbatzoudis, 2005) }\end{array}$ & $\begin{array}{l}-366 \text { adolesc. } \\
-14.2 \text { años } \\
(\mathrm{SD}=0.7) \\
\text { - Grecia }\end{array}$ & $\begin{array}{l}-12 \text { semanas } \\
\text { - CT } \\
\text { - I }(2 \text { centros, } 195) \\
\text { C }(2,171)\end{array}$ & $\begin{array}{l}\text { Programa educativo desarrollado en EF a través de } \\
\text { exposiciones y debates sobre el cambio de } \\
\text { comportamiento relacionado con AF. Se } \\
\text { proporciona información sobre oportunidades de } \\
\text { AF dentro y fuera de la escuela. }\end{array}$ \\
\hline $\begin{array}{l}\text { Pate (2005) } \\
\text { LEAP }{ }_{\text {2005) }}^{\text {(Pate et al., }}\end{array}$ & $\begin{array}{l}\text { - } 2744 \text { chicas } \\
-13.6(\mathrm{SD}=0.6) \\
\text { años } \\
\text { - USA }\end{array}$ & $\begin{array}{l}-1 \text { año } \\
\text { - RCT } \\
\text { - I ( } 12 \text { escuelas, } \\
1221 \text { chicas }) ; C(12, \\
1523)\end{array}$ & $\begin{array}{l}\text { Los componentes de esta intervención basada en el } \\
\text { modelo socioecológico son: clases de EF solo para } \\
\text { chicas, un componente educativo, la creación de un } \\
\text { ambiente escolar favorable, la implicación del } \\
\text { profesorado y la familia y vínculos con la } \\
\text { comunidad. }\end{array}$ \\
\hline $\begin{array}{l}\text { Bayne-Smith } \\
\text { (2004) } \\
\text { PATH }{ }_{\text {et al., 2004) }}^{(\text {Bayne-Smith }}\end{array}$ & $\begin{array}{l}\text { - } 442 \text { chicas } \\
\text { - } \approx 16 \text { años } \\
\text { - USA }\end{array}$ & $\begin{array}{l}\text { - } 12 \text { semanas } \\
\text { - RCT } \\
\text { - I ( } 310 \text { chicas }), C \\
(132)\end{array}$ & $\begin{array}{l}\text { Clases de EF } 5 \text { días a la semana que consta de AF } \\
\text { vigorosa y un currículum educativo sobre temáticas } \\
\text { relacionadas con la salud cardiovascular y la } \\
\text { condición física. }\end{array}$ \\
\hline $\begin{array}{c}\text { Jamner (2004) } \\
\text { Project FAB } \\
\text { (Jamner et al., 2004) }\end{array}$ & $\begin{array}{l}\text { - } 58 \text { chicas } \\
\text { sedentarias } \\
\text { - } 14-16 \text { años } \\
\text { - USA }\end{array}$ & $\begin{array}{l}\text { - } 4 \text { meses } \\
\text { - CT } \\
\text { - I (1 school, } 25 \\
\text { chicas); } C(1,22)\end{array}$ & $\begin{array}{l}\text { Clases de EF } 5 \text { días a la semana que consta de } \\
\text { actividades físicas variadas e innovadoras y un } \\
\text { componente educativo ( } 1 \text { día/semana), sobre } \\
\text { beneficios de la AF y estrategias para ser activa. }\end{array}$ \\
\hline $\begin{array}{l}\text { Simon (2004) } \\
\text { ICAPS (Simon et al., } \\
\text { 2004) }\end{array}$ & $\begin{array}{l}-954 \text { adolesc. } \\
-11.7 \text { años } \\
(\mathrm{SD}=0.6) \\
\text { - Francia }\end{array}$ & $\begin{array}{l}\text { - } 4 \text { años } \\
\text { - RCT } \\
\text { - I ( } 4 \text { centros, } 475 \\
\text { estud.); C }(4,479)\end{array}$ & $\begin{array}{l}\text { Consta de un componente educativo integrado en } \\
\text { diferentes asignaturas (EF entre ellas), nuevas } \\
\text { oportunidades para la AF durante el horario escolar } \\
\text { y extraescolar y reuniones con padres y profesores } \\
\text { para fomentar el apoyo social. }\end{array}$ \\
\hline $\begin{array}{c}\text { Neumark- } \\
\text { Sztainer (2003) } \\
\text { New Moves } \\
\text { (Neumark-Sztainer et } \\
\text { al., 2003) }\end{array}$ & $\begin{array}{l}\text { - } 201 \text { chicas } \\
\text { inactivas } \\
-15.4 \text { años } \\
(\mathrm{SD}=1.1) \\
\text { - USA }\end{array}$ & $\begin{array}{l}\text { - } 16 \text { semanas } \\
\text { - RCT } \\
\text { - I }(3 \text { centros, } 89 \\
\text { chicas }) \text {; }(3,112)\end{array}$ & $\begin{array}{l}\text { Clases de EF solo para chicas (4 días/semana) con } \\
\text { AF adecuadas a chicas de diferentes niveles de } \\
\text { competencia motriz y sesiones educativas con } \\
\text { debates sobre apoyo social y nutrición. Se envían } \\
\text { cartas a los padres y se crean vínculos con la } \\
\text { comunidad. }\end{array}$ \\
\hline $\begin{array}{c}\text { Sallis (2003) } \\
\text { M-SPAN (Sallis et } \\
\text { al., 2003) }\end{array}$ & $\begin{array}{l}-24 \text { centros } \\
(1109 \text { adolesc. } \\
\text { /centro }) \\
-11-13 \text { años } \\
\text { - USA }\end{array}$ & $\begin{array}{l}\text { - } 2 \text { años } \\
\text { - RCT } \\
\text { - I (12 centros }), C \\
\text { ( } 12 \text { centros })\end{array}$ & $\begin{array}{l}\text { Clases de EF diarias, con formación previa del } \\
\text { profesorado para ofrecer una EF de calidad. Se } \\
\text { incrementan las oportunidades para la AF en la } \\
\text { escuela ofreciéndose financiación para nuevos } \\
\text { materiales y programas de AF. Se implica a los } \\
\text { propios estudiantes y a los padres. }\end{array}$ \\
\hline
\end{tabular}




\begin{tabular}{|c|c|c|c|}
\hline $\begin{array}{l}\text { Gortmaker } \\
\text { (1999) } \\
\text { Planet } \\
\text { Health (Gortmaker } \\
\text { et al., 1999) }\end{array}$ & $\begin{array}{l}-1295 \text { adolesc. } \\
\text { - } \approx 11.7 \text { años } \\
\text { - USA }\end{array}$ & $\begin{array}{l}-2 \text { años } \\
\text { - RCT } \\
\text { - I ( } 5 \text { centros }), \text { C ( } 5 \\
\text { centros })\end{array}$ & $\begin{array}{l}\text { Currículum que consta de } 32 \text { sesiones educativas } \\
\text { impartidas en EF y otras áreas por el profesorado } \\
\text { del centro y que se orientan a incrementar los } \\
\text { niveles de AF y mejorar la alimentación. }\end{array}$ \\
\hline
\end{tabular}

RCT: Randomized Controlled Trial (estudio experimental). MPS: Modelo de Promoción de la Salud; TTM: Modelo Transteórico o de los Estados de Cambio del Comportamiento; I: Grupo de intervención; C: Grupo de control.

Tabla 1. Síntesis de los programas de intervención de promoción de la AF salud en la adolescencia que incorporan actuaciones en la EF escolar.

Características generales de los programas de promoción de la AF orientada hacia la salud que integran la EF curricular.

11 de los 15 programas de intervención se han desarrollado en los Estados Unidos (Bayne-Smith et al., 2004; Gortmaker et al., 1999; Jamner et al., 2004; Jones et al., 2008; McMurray et al., 2009b; Neumark-Sztainer et al., 2003; Neumark-Sztainer et al., 2010; Pate et al., 2005; Sallis et al., 2003; Webber et al., 2008; Young et al., 2006), uno en Irán (Taymoori et al., 2008) y tres en Europa, concretamente en Francia (Simon et al., 2004), Holanda (Singh et al., 2006) y Grecia (Tsorbatzoudis, 2005).

De estos programas, 5 tienen como objetivo prioritario incrementar los niveles de AF de los participantes (Pate et al., 2005; Taymoori et al., 2008; Tsorbatzoudis, 2005; Webber et al., 2008; Young et al., 2006), mientras que otros se centran en la prevención de la obesidad (Gortmaker et al., 1999; Neumark-Sztainer et al., 2003; Neumark-Sztainer et al., 2010; Sallis et al., 2003; Singh et al., 2006) o diabetes (McMurray et al., 2009b), mejorar la densidad ósea (Jamner et al., 2004; Jones et al., 2008) y la prevención de las enfermedades cardiovasculares (Bayne-Smith et al., 2004; Simon et al., 2004). De estos programas es interesante señalar que 8 se centran de forma exclusiva en la promoción de la AF (Jamner et al., 2004; Jones et al., 2008; Pate et al., 2005; Taymoori et al., 2008; Tsorbatzoudis, 2005; Webber et al., 2008; Young et al., 2006), mientras que los 7 programas restantes tienen un carácter más integral e incluyen también estrategias relacionadas con la adopción de una alimentación equilibrada y saludable (Gortmaker et al., 1999; McMurray et al., 2009b; NeumarkSztainer et al., 2003; Neumark-Sztainer et al., 2010; Sallis et al., 2003; Simon et al., 2004; Singh et al., 2006).

En relación con la población a la que se dirigen, 6 programas se orientan al colectivo de chicos y chicas adolescentes en su conjunto (Gortmaker et al., 1999; McMurray et al., 2009b; Sallis et al., 2003; Simon et al., 2004; Singh et al., 2006; Tsorbatzoudis, 2005), mientras que, en un intento de abordar la problemática de las diferencias de género en relación a los niveles de AF, 9 programas de intervención están dirigidos específicamente a las chicas (Bayne-Smith et al., 2004; Jamner et al., 2004; Jones et al., 2008; Neumark-Sztainer et al., 2003; Neumark-Sztainer et al., 2010; Pate et al., 2005; Taymoori et al., 2008; Webber et al., 2008; Young et al., 2006). Las 
chicas sedentarias (Neumark-Sztainer et al., 2003; Neumark-Sztainer et al., 2010; Taymoori et al., 2008), propensas a la obesidad (Neumark-Sztainer et al., 2003; Neumark-Sztainer et al., 2010) o con un bajo nivel de condición física (Jamner et al., 2004) constituyen una población de riesgo en la que se centran este tipo de intervenciones. No obstante, dos de estos programas adoptan un carácter inclusivo $\mathrm{y}, \mathrm{a}$ pesar de que la intervención se dirige a las chicas, se incluye a los chicos como parte del contexto escolar en el que se desarrolla el conjunto del programa de intervención (Pate et al., 2005; Webber et al., 2008).

Las intervenciones tienen una duración muy variable, que oscila entre las 12 semanas de los trabajos de Bayne-Smith (2004), y Tsorbatzoudis (2005), hasta los 4 años del programa ICAPS (Simon et al., 2004), mientras que los programas HEALTHY study (McMurray et al., 2009b), TAAG (Webber et al., 2008), M-SPAM (Sallis et al., 2003) y Planet Health (Gortmaker et al., 1999) fueron implementados durante dos años.

En cuanto al diseño de la intervención, excepto un estudio de carácter cuasiexperimental (Tsorbatzoudis, 2005), el resto de las intervenciones utilizan diseños experimentales, bien por conglomerados o por individuos (Bayne-Smith et al., 2004; Young et al., 2006), aunque en la mayoría de ellos no se especifica o no se describe adecuadamente el procedimiento para realizar la asignación aleatoria de los participantes al grupo de intervención o de control $(\mathrm{n}=9)$. Otras limitaciones de estos trabajos es el uso de instrumentos de escasa fiabilidad y validez $(n=4)$, la ausencia de "blinding" o intervención a ciegas $(\mathrm{n}=10)$ y los elevados índices de abandono de la intervención por los participantes $(\mathrm{n}=4)$.

\section{La EF escolar en los programas de promoción de la AF-salud.}

En la Tabla 2 se muestra el carácter de la EF escolar dentro de los programas de intervención, es decir, el papel que desempeña esta área curricular en el conjunto de las intervenciones orientadas a promocionar la AF-salud en la adolescencia. Seis de estos programas se desarrollan exclusivamente en las clases de EF (Bayne-Smith et al., 2004; Gortmaker et al., 1999; Jamner et al., 2004; Jones et al., 2008; McMurray et al., 2009a; Tsorbatzoudis, 2005), por lo que integran acciones dirigidas únicamente a los destinatarios de la intervención. Los otros cuatro programas incorporan además actuaciones en el entorno social próximo con el propósito de implicar a las familias como el apoyo social clave que necesitan los adolescentes para adoptar estilos de vida activos. Básicamente se trata de reuniones o cartas informativas destinadas a los padres (Neumark-Sztainer et al., 2003; Taymoori et al., 2008; Young et al., 2006), eventos de AF de las participantes con las madres (Taymoori et al., 2008) o bolsas con material educativo para que los participantes lleven a casa (Neumark-Sztainer et al., 2010). En el conjunto de estos 10 programas en los que la EF se configura como el componente clave de la intervención, las clases de EF son exclusivamente para las chicas en seis programas (Jamner et al., 2004; Jones et al., 2008; Neumark-Sztainer et al., 2003; Neumark-Sztainer et al., 2010; Taymoori et al., 2008; Young et al., 2006). 


\begin{tabular}{|c|c|}
\hline \multicolumn{2}{|c|}{ Carácter de la EF escolar dentro de los programas de intervención } \\
\hline Componente exclusivo de la intervención & $\begin{array}{l}\text { Número de estudios }{ }^{\text {(ref. principal) }} \\
6^{\text {(Bayne-Smith et al., 2004; Gortmaker et al., 1999; }} \\
\text { Jamner et al., 2004; Jones et al., 2008; McMurray et al., } \\
\text { 2009a; Tsorbatzoudis, 2005) }\end{array}$ \\
\hline $\begin{array}{l}\text { Principal componente de la intervención, que } \\
\text { integra actuaciones complementarias }\end{array}$ & $\begin{array}{l}4_{\text {(Neumark-Sztainer et al., 2003; Neumark-Sztainer et }} \\
\text { al., 2010; Taymoori et al., 2008; Young et al., 2006) }\end{array}$ \\
\hline $\begin{array}{l}\text { Es un componente más del programa, que } \\
\text { integra componentes en otros ámbitos de } \\
\text { actuación }\end{array}$ & $\begin{array}{l}5^{\text {(McMurray et al., 2009b; Pate et al., 2005; Sallis et al., }} \\
\text { 2003; Simon et al., 2004; Webber et al., 2008) }\end{array}$ \\
\hline
\end{tabular}

Sólo práctica de actividades físicas

Sólo componente educativo (Dirigidas al grupoclase o desarrolladas individualmente con cada estudiante)

Ambos componentes
Número de estudios (ref. principal)

4 (Pate et al., 2005; Sallis et al., 2003; Webber et al., 2008) (McMurray et al., 2009b)

4 (Gortmaker et al., 1999; Simon et al., 2004; Singh et al., 2006; Taymoori et al., 2008)

7 (Bayne-Smith et al., 2004; Jamner et al., 2004; Jones et al., 2008; Neumark-Sztainer et al., 2003; NeumarkSztainer et al., 2010; Tsorbatzoudis, 2005; Young et al., 2006)

Actuaciones del profesorado de EF en la intervención desarrollada en la EF curricular Número de estudios (principal ref.)

Permanece ajeno al desarrollo del programa de 2 (Taymoori et al., 2008; Young et al., 2006) intervención

Implementa un currículum cerrado, previamente definido en el programa de intervención

10 (Bayne-Smith et al., 2004; Gortmaker et al., 1999; Jamner et al., 2004; Jones et al., 2008; McMurray et al., 2009b; Neumark-Sztainer et al., 2003; Neumark-Sztainer et al., 2010; Simon et al., 2004; Singh et al., 2006; Tsorbatzoudis, 2005)

Recibe formación para adaptar el currículum y su enseñanza según las directrices del programa 3 (Pate et al., 2005; Sallis et al., 2003; Webber et al., 2008) de intervención

Tabla 2. La Educación Física escolar en los programas de promoción de la AF-salud.

En los otros cinco programas, la EF se configura como un componente más de la intervención, dentro de programas de carácter más holístico que incorporan actuaciones en el entorno próximo de los jóvenes, orientadas no sólo a promover el apoyo social, sino también a incrementar sus oportunidades para ser activos tanto en el centro escolar como en la comunidad (McMurray et al., 2009b; Pate et al., 2005; Sallis et al., 2003; Simon et al., 2004; Webber et al., 2008). Generalmente se ofrece apoyo logístico y/o financiación a las escuelas para que oferten nuevos programas y 
materiales de AF (McMurray et al., 2009b; Sallis et al., 2003; Simon et al., 2004). Otras intervenciones tratan de fomentar los vínculos entre la escuela y la comunidad para proporcionar a los jóvenes más opciones para la práctica de AF (Pate et al., 2005; Webber et al., 2008).

Profundizando en los componentes de la EF curricular en los programas de intervención (Tabla 2), la mayor parte de estos programas combinan la práctica de actividades físicas junto con un componente educativo orientado a que los estudiantes adquieran las habilidades comportamentales necesarias para adoptar un estilo de vida activo. El estudio de Jones (2008), que se orienta a la promoción de la salud ósea de las chicas, consiste en 10 minutos de actividades de alto impacto integradas en el calentamiento de las clases de EF ordinarias, junto con un componente educativo orientado a promover la práctica de este tipo de actividades físicas. Integrar la práctica de diferentes actividades físicas junto con un componente educativo es el esquema básico que siguen los estudios de Jamner et al. (2004) y de Bayne-Smith et al. (2004). No obstante, en el primer caso, el componente educativo se orienta específicamente a la práctica de $\mathrm{AF}$, y en el segundo caso a promover la salud cardiovascular y la condición física. El estudio de Tsorbatzoudis et al. (2005) consiste en un programa educativo de 12 semanas de duración desarrollado a través de pequeñas conferencias y debates sobre la AF y la salud; además se proporciona información al alumnado sobre las oportunidades para practicar AF fuera del horario curricular. Los programas Life Skills (Young et al., 2006) y New Moves (Neumark-Sztainer et al., 2003; NeumarkSztainer et al., 2010), se caracterizan por desarrollar actividades físicas innovadoras y orientadas al disfrute, sobre todo orientadas a implicar a los estudiantes con menor nivel de habilidad o condición física. Ambos programas tienen un componente educativo, centrados básicamente en proporcionar información sobre los beneficios de la vida activa, la resolución de problemas y cómo obtener el apoyo social necesario.

Cuatro programas desarrollan en la EF escolar un componente educativo exclusivamente (Gortmaker et al., 1999; Simon et al., 2004; Singh et al., 2006; Taymoori et al., 2008) que se desarrolla a través de sesiones formativas con el grupo clase. No obstante, dos programas incorporan además sesiones de carácter individual, que en el programa DoIT (Singh et al., 2006) se implementan mediante un programa informático y que en la intervención de Taymoori et al. (2008) consisten en sesiones individualizadas centradas en ayudar a las participantes a establecerse sus propias metas de $\mathrm{AF}$ y a realizar el cambio de comportamiento necesario para conseguirlas.

La práctica de AF es el componente exclusivo de las clases de EF en cuatro programas (McMurray et al., 2009b; Pate et al., 2005; Sallis et al., 2003; Webber et al., 2008). En todos los casos, se trata de intervenciones de carácter socioecológico orientadas a lograr cambios positivos en el entorno social, físico y organizativo escolar para impulsar la práctica de AF y que desarrollan el componente de la EF escolar a través de la formación del profesorado de EF.

En su conjunto, el componente de práctica de $\mathrm{AF}$ de estos programas se centra en incrementar los niveles de práctica de AF durante las clases, priorizando el disfrute con 
la propia actividad y la autoeficacia, especialmente en el caso de los/as menos habilidosos. Algunos ejemplos de estrategias utilizadas son ofertar un amplio rango de actividades no competitivas e innovadoras (e.g. capoeira, kick-boxing, marchas con podómetros), (Jamner et al., 2004; Neumark-Sztainer et al., 2003; Neumark-Sztainer et al., 2010; Pate et al., 2005; Webber et al., 2008; Young et al., 2006), ofrecer la posibilidad de elegir entre diferentes alternativas (Webber et al., 2008), juegos y actividades en pequeños grupos (McMurray et al., 2009b; Pate et al., 2005; Webber et al., 2008; Young et al., 2006), modificación de las reglas de los juegos y deportes para hacerlos más participativos (Webber et al., 2008) y la provisión de material deportivo suficiente y no convencional (McMurray et al., 2009b; Sallis et al., 2003; Webber et al., 2008).

Dentro de la asignatura curricular de EF resulta relevante conocer cuáles son las actuaciones del profesorado de EF en la intervención desarrollada en la EF curricular (Tabla 2), puesto que es el agente que habitualmente imparte esta materia en los centros escolares.

En la mayoría de las intervenciones, el profesorado de EF es el encargado de implementar un currículum cerrado y diseñado por los investigadores (Bayne-Smith et al., 2004; Gortmaker et al., 1999; Jamner et al., 2004; Jones et al., 2008; NeumarkSztainer et al., 2003; Neumark-Sztainer et al., 2010; Taymoori et al., 2008; Tsorbatzoudis, 2005; Young et al., 2006). No obstante, en el programa New Moves (Neumark-Sztainer et al., 2003; Neumark-Sztainer et al., 2010) se tienen en cuenta las opiniones recogidas de los estudiantes y del profesorado de EF a través de una extensa evaluación formativa. Por otra parte, el profesorado de EF participante en el programa HEALTHY (McMurray et al., 2009b) recibe formación para implementar las actividades que integran el programa configurando las sesiones de EF, así como la asistencia de un profesor ayudante para el desarrollo de las clases.

En dos programas el profesorado de EF permanece ajeno al desarrollo de la intervención, que es implementada por un profesor contratado específicamente para ello (Young et al., 2006) o por los propios investigadores (Taymoori et al., 2008).

La formación del profesorado de EF para adaptar el currículum y su enseñanza a las directrices del programa de intervención es el enfoque adoptado por los tres programas de carácter socioecológico (Pate et al., 2005; Sallis et al., 2003; Webber et al., 2008). Dicha formación consiste, entre otros aspectos, en estrategias para el control de la clase, actividades específicas para el desarrollo de habilidades motrices e incrementar los niveles de AF, cómo proporcionar a los estudiantes la posibilidad de elegir, etc.

\section{Discusión}

A través de la presente revisión se pone de manifiesto la conveniencia de que las intervenciones de promoción de la AF orientada hacia la salud en la edad adolescente incorporen la EF escolar como uno de sus elementos clave. Dentro de la promoción de 
la salud escolar, este enfoque contribuiría a revalorizar la aportación única y específica de la EF dentro del currículum educativo.

No obstante, dos cuestiones se revelan como claves para que pueda desempeñar adecuadamente esta función. En primer lugar, sería necesario incrementar la asignación de horas que posee dentro del currículum, puesto que sus propósitos de promoción de la AF-salud están seriamente condicionando por el reducido tiempo del que se dispone para desarrollar sus enseñanzas. Específicamente, desde el Parlamento Europeo se insta a que el horario escolar garantice al menos tres horas de EF por semana en la Enseñanza Secundaria (Parlamento Europeo, 2007), situación que dista de la realidad educativa en nuestro país. En segundo lugar, y en coherencia con el modelo socioecológico de promoción de la salud (Elder et al., 2007; Sallis et al., 2008), es fundamental que la EF desempeñe esta función dentro de iniciativas de carácter más amplio que integren actuaciones dirigidas al entorno social, físico y organizativo del centro escolar.

De esta forma, se configuraría un entorno apropiado que, desde diferentes actuaciones, se orientaría al mismo objetivo de promoción de la AF-salud de los escolares. Así sería posible, por ejemplo, que el componente educativo que implementan los programas analizados, y que tiene un valor indiscutible al proporcionar a los estudiantes los recursos y habilidades necesarios para adoptar un estilo de vida activo, se desarrollen de forma complementaria por otras áreas curriculares, dedicándose la clase de EF de forma exclusiva a la práctica de actividades físicas.

Como se desprende de la revisión de programas realizada, estas actividades físicas se han de orientar principalmente a fomentar el disfrute con la propia actividad y los sentimientos de autoeficacia, aspectos que se señalan como claves para implicarse en la práctica de actividades físicas (Dishman et al., 2004; Dishman et al., 2005; Schneider \& Cooper, 2011). Unos niveles de AF adecuados, priorizando las actividades físicas de intensidad moderada a vigorosa (Fairclough \& Stratton, 2005), son también fundamentales de cara a contribuir de forma significativa al cumplimiento de las recomendaciones de AF en la adolescencia.

Considerando los resultados obtenidos, se confirma que el profesorado de EF se perfila como el agente clave de este tipo de intervenciones. Aunque en la mayoría de los programas analizados el profesorado implementa un currículum cerrado, una forma eficaz de implicarlo de forma activa en el desarrollo de este tipo de intervenciones y de asegurar la sostenibilidad en el tiempo de la intervención, es la creación en el centro escolar de una estructura organizativa, liderada por un profesor/a de EF comprometido, que haga suyo el programa y que lo adapte a las características de cada contexto escolar (Ward et al., 2006). Como contrapartida puede resultar que en la práctica se trate de programas de características muy diversas respecto al diseño inicial. No obstante, se puede hacer compatible la adaptabilidad y flexibilidad de la intervención con su fidelidad, siguiendo estrategias como las siguientes: proporcionar al profesorado una formación intensa y adecuada así como asistencia técnica durante el desarrollo del 
programa; priorizar y seleccionar dentro de un programa flexible aquellos elementos esenciales de la intervención; y finalmente, establecer un buen proceso de evaluación de la implementación (Saunders et al., 2006).

En conclusión, la EF escolar desempeña un papel clave en la promoción de la $\mathrm{AF}$ entre los jóvenes, tarea compleja debido a las dificultades asociadas con el diseño, implementación y evaluación de este tipo de intervenciones, pero que compensa si se consideran los beneficios para la salud que se derivan de tener una población de escolares físicamente activos.

\section{Referencias bibliográficas}

AHRABI-FARD, I., \& MATVIENKO, O. A. (2005). Promoción de una educación activa de la actividad física orientada a la salud en las clases de Educación Física. Cultura, Ciencia y Deporte, 1:3, pp. 163-170.

BAYNE-SMITH, M., FARDY, P. S., AZZOLLINI, A., MAGEL, J., SCHMITZ, K. H., AGIN, D., BAYNE-SMITH, M., FARDY, P. S., AZZOLLINI, A., MAGEL, J., SCHMITZ, K. H., \& AGIN, D. (2004). Improvements in heart health behaviors and reduction in coronary artery disease risk factors in urban teenaged girls through a school-based intervention: the PATH program. American Journal of Public Health, 94:9, pp. 1538-1543.

BUTCHER, K., SALLIS, J. F., MAYER, J. A., \& WOODRUFF, S. (2008). Correlates of physical activity guidelines compliance for adolescents in 100 US cities. Journal of Adolescent Health, 42:4, pp. 360-368.

CURRIE, C., GABHAINN, S. N., GODEAU, E., ROBERTS, C., SMITH, R., CURRIE, D., PICKET, W., RICHTER, M., MORGAN, A., \& BARNEKOW, V. (2008). Inequalities in young people's health. Health Behavior in School Aged Children (HBSC) study: International report from the 2005/2006 survey. Copenhagen: WHO Regional Office for Europe. Disponible en: http://www.euro.who.int/en/what-we-do/health-topics/Life-stages/child-andadolescent-health/publications2/2011/inequalities-in-young-peoples-health.-hbscinternational-report-from-the-20052006-survey. Fecha consulta: Dic. 2010.

DE MEESTER, F., VAN LENTHE, F., SPITTAELS, H., LIEN, N., \& DE BOURDEAUDHUIJ, I. (2009). Interventions for promoting physical activity among European teenagers: a systematic review. International Journal of Behavioral Nutrition and Physical Activity, 6:1, p. 82.

DISHMAN, R. K., MOTL, R. W., SAUNDERS, R., FELTON, G., WARD, D. S., DOWDA, M., \& PATE, R. R. (2004). Self-efficacy partially mediates the effect of a school-based physical-activity intervention among adolescent girls. Preventive Medicine, 38:5, pp. 628-636. 
DISHMAN, R. K., MOTL, R. W., SAUNDERS, R., FELTON, G., WARD, D. S., DOWDA, M., \& PATE, R. R. (2005). Enjoyment mediates effects of a schoolbased physical-activity intervention. Medine in Science \& Sports Exercise, 37:3, pp. 478-487. doi: 10.1016/j.ypmed.2003.12.007

EldER, J. P., LYTLE, L. A., SAlliS, J. F., YOUNG, D. R., STECKLER, A., SIMMONS-MORTON, D. G., STONE, E., JOBE, J. B., STEVENS, J., LOHMAN, T., WEBBER, L. S., PATE, R., SAKSVIG, B. I., \& RIBISL, K. M. (2007). A description of the social-ecological framework used in the trial of activity for adolescent girls (TAAG). Health Education Research, 22:2, pp. 155-165.

ESTRADA, C., CRUZ, J.L., AGUIRRE, R. (DIRS.). (2010). Factores socioculturales que influyen en la práctica de actividad física en la infancia y adolescencia en la Comunidad de Madrid. Madrid: Dirección General de Atención Primaria. Servicio de Promoción de la Salud y Prevención.

FAIRCLOUGH, S., \& STRATTON, G. (2005). "Physical education makes you fit and healthy". Physical education's contribution to young people's physical activity levels. Health Education Research, 20:1, pp. 14-23.

FERNÁNDEZ, E., CAMACHO, M. J., SIERRA, M. A., VÁZQUEZ, B., RODRÍGUEZ, M. I., MENDIZÁBAL, S., SÁNCHEZ, F., \& SÁNCHEZ, M. (2007). Estudio de los estereotipos de género vinculados con la actividad física y el deporte en los centros docentes de Educación Primaria y Secundaria. Infome de investigación: Instituto de la Mujer.

FERNÁNDEZ, E., VÁZQUEZ, B., CAMACHO, M. J., SÁNCHEZ, F., MARTÍNEZ, O., RODRÍGUEZ, M. I., \& AZNAR, S. (2007). La inclusión de la actividad física y el deporte en el estilo de vida de las mujeres adolescentes: estudio de los factores clave y pautas de intervención. Investigaciones en Ciencias del Deporte, 46 pp. 23-60.

GORDON-LARSEN, P., NELSON, M. C., \& POPKIN, B. M. (2004). Longitudinal physical activity and sedentary behavior trends: adolescence to adulthood. American Journal of Preventive Medicine, 31:4, pp. 353-353.

GORTMAKER, S. L., PETERSON, K., WIECHA, J., SOBOL, A. M., DIXIT, S., FOX, M. K., \& LAIRD, N. (1999). Reducing obesity via a school-based interdisciplinary intervention among youth - Planet health. Archives of Pediatrics \& Adolescent Medicine, 153:4, pp. 409-418.

HARDMAN, K. (2003). An up-date on the status of Physical Education in Schools Worldwide: technical report for the World Health Organisation. Geneva: World Health Organisation. Disponible en: http://www.icsspe.org/documente/PEworldwide.pdf. Fecha de consulta: Enero 2009.

ICSSPE. (2005). Magglingen commitment for Physical Education. Magglingen, Switzerland.

JAGO, R., \& BARANOWSKI, T. (2004). Non-curricular approaches for increasing physical activity in youth: a review. Preventive Medicine, 39:1, pp. 157-163. 
JAMNER, M. S., SPRUIJT-METZ, D., BASSIN, S., \& COOPER, D. M. (2004). A controlled evaluation of a school-based intervention to promote physical activity among sedentary adolescent females: Project FAB. Journal of Adolescent Health, $34: 4$, pp. 279-289.

JANSSEN, I., \& LEBLANC, A. (2010). Systematic review of the health benefits of physical activity and fitness in school-aged children and youth. International Journal of Behavioral Nutrition and Physical Activity, 7:1, p. 40. doi:10.1186/14795868-7-40

JONES, D., HOELSCHER, D. M., KELDER, S. H., HERGENROEDER, A., \& SHARMA, S. V. (2008). Increasing physical activity and decreasing sedentary activity in adolescent girls - The Incorporating More Physical Activity and Calcium in Teens (IMPACT) study. International Journal of Behavioral Nutrition and Physical Activity, 5 p. 42.

KAHN, E., RAMSEY, L. T., BROWNSON, R. C., HEALTH, G. W., HOWZE, E. H., POWELL, K. E., STONE, E. J., RAJAB, M. W., CORSO, P., \& SERVICES, T. F. O. C. P. (2002). The effectiveness of interventions to increase physical activity. A systematic review. American Journal of Preventive Medicine, 22:4S, pp. 73-107.

KAHN, J. A., HUANG, B., GILlman, M. W., FIELD, A. E., AUSTIN, S. B., COLDITZ, G. A., \& FRAZIER, A. L. (2008). Patterns and determinants of physical activity in US adolescents. Journal of Adolescent Health, 42:4, pp. 369-377.

KIMM, S. Y. S., GLYNN, N. W., KRISKA, A. M., FITZGERALD, S. L., AARON, D. J., SIMILO, S. L., MCMAHON, R. P., \& BARTON, B. A. (2000). Longitudinal changes in physical activity in a biracial cohort during adolescence. Medicine and Science in Sports and Exercise, 32:8, pp. 1445-1454.

LUBANS, D. R., FOSTER, C., \& BIDDLE, S. J. H. (2008). A review of mediators of behavior in interventions to promote physical activity among children and adolescents. Preventive Medicine, 47:5, pp. 463-470.

LUBANS, D. R., MORGAN, P. J., \& TUDOR-LOCKE, C. (2009). A systematic review of studies using pedometers to promote physical activity among youth. Preventive Medicine, 48:4, pp. 307-315.

MCKENZIE, T. L., \& LOUNSBERY, M. A. F. (2009). School Physical Education: The Pill Not Taken. American Journal of Lifestyle Medicine, 3:3, pp. 219-225. doi: $10.1177 / 1559827609331562$

MCMURRAY, R. G., BASSIN, S., JAGO, R., BRUECKER, S., MOE, E. L., MURRAY, T., MAZZUTO, S. L., \& VOLPE, S. L. (2009a). HEALTHY study rationale, design and methods: moderating risk of type 2 diabetes in multi-ethnic middle school students. International Journal of Obesity, 33 pp. S4-S20. doi:10.1038/ijo.2009.112 
MCMURRAY, R. G., BASSIN, S., JAGO, R., BRUECKER, S., MOE, E. L., MURRAY, T., MAZZUTO, S. L., \& VOLPE, S. L. (2009b). Rationale, design and methods of the HEALTHY study physical education intervention component. International Journal of Obesity, 33 pp. S37-S43. doi:10.1038/ijo.2009.114

NASPE. (2011). Physical Education is critical to educating the whole child. Reston, VA.: National Association for Sport and Physical Education. Disponible en: http://www.aahperd.org/naspe/standards/upload/Physical-Education-Is-Critical-toEducating-the-Whole-Child-Final-5-19-2011.pdf. Fecha consulta: Junio 2011.

NEUMARK-SZTAINER, D., STORY, M., HANNAN, P. J., \& REX, J. (2003). New Moves: a school-based obesity prevention program for adolescent girls. Preventive Medicine, 37:1, pp. 41-51.

NEUMARK-SZTAINER, D. R., FRIEND, S. E., FLATTUM, C. F., HANNAN, P. J., STORY, M. T., BAUER, K. W., FELDMAN, S. B., \& PETRICH, C. A. (2010). New Moves-Preventing Weight-Related Problems in Adolescent Girls: A GroupRandomized Study. American Journal of Preventive Medicine, 39:5, pp. 421-432.

PARLAMENTO EUROPEO. (2007). Resolución del Parlamento Europeo, de 13 de noviembre de 2007, sobre la función del deporte en la educación: Parlamento Europeo.

PATE, R. R., DAVIS, M. G., ROBINSON, T. N., STONE, E. J., MCKENZIE, T. L., \& YOUNG, J. C. (2006). Promoting physical activity in children and youth: a leadership role for schools. Circulation, 114:11, pp. 1214-1224.

PATE, R. R., FREEDSON, P. S., SALLIS, J. F., W.C.TAYLOR, SIRARD, J., TROST, S. G., \& DOWDA, M. (2002). Compliance with physical activity guidelines: prevalence in a population of children and youth. Annals of Epidemiology, 12 pp. 303-308.

PATE, R. R., WARD, D. S., O'NEILL, J. R., \& DOWDA, M. (2007). Enrollment in physical education is associated with overall physical activity in adolescent girls. Research Quarterly for Exercise and Sport, 78:4, pp. 265-270.

PATE, R. R., WARD, D. S., SAUNDERS, R. P., FELTON, G., DISHMAN, R. K., \& DOWDA, M. (2005). Promotion of Physical Activity Among High-School Girls: A Randomized Controlled Trial. American Journal of Public Health, 95:9, pp. 15821587. doi: 10.2105/AJPH.2004.045807

RIDDOCH, C. J., \& ANDERSEN, L. (2004). Physical activity levels and patterns of 9-15yr-old European children. Medicine \& Science in Sport \& Exercise 36:1, pp. 86-92.

ROMÁN, B., SERRA-MAJÉM, L., RIBAS-BARBA, L., PÉREZ-RODRIGO, C., \& ARANCETA, J. (2008). How many children and adolescents in Spain comply with the recommendations on physical activity? Journal of Sports Medicine and Physical Fitness, 48:3, pp. 380-387. 
SALLIS, J. F. (2000). Age-related decline in physical activity: a synthesis of human and animal studies. Medicine and Science in Sports and Exercise, 32:9, pp. 1598-1600.

SALLIS, J. F., MCKENZIE, T. L., CONWAY, T. L., ELDER, J. P., PROCHASKA, J. J., BROWN, M., ZIVE, M. M., MARSHALL, S. J., \& ALCARAZ, J. E. (2003). Environmental interventions for eating and physical activity - A randomized controlled trial in middle schools. American Journal of Preventive Medicine, 24:3, pp. 209-217. doi: 10.1016/S0749-3797(02)00646-3

SALLIS, J. F., OWEN, N., \& FISHER, E. (2008). Ecological Models of Health Behavior. In K. Glanz, B. Rimer \& K. Viswanath (Eds), Health Behavior and Health Education: Theory, Research, and Practice (pp. 465-482). United States: Jossey-Bass.

SAUNDERS, R. P., WARD, D., FELTON, G. M., DOWDA, M., \& PATE, R. R. (2006). Examining the link between program implementation and behavior outcomes in the lifestyle education for activity program (LEAP). Evaluation and Program Planning, 29:4, pp. 352-364.

SCHNEIDER, M., \& COOPER, D. (2011). Enjoyment of exercise moderates the impact of a school-based physical activity intervention. International Journal of Behavioral Nutrition and Physical Activity, 8:1,p. 64. doi:10.1186/1479-5868-8-64

SIMON, C., WAGNER, A., DIVITA, C., RAUSCHER, E., KLEIN-PLATAT, C., ARVEILER, D., SCHWEITZER, B., \& TRIBY, E. (2004). Intervention centred on adolescents' physical activity and sedentary behaviour (ICAPS): concept and 6 month results. International Journal of Obesity, 28 pp. S96-S103.

SINGH, A. S., PAW, M., KREMERS, S. P. J., VISSCHER, T. L. S., BRUG, J., \& VAN MECHELEN, W. (2006). Design of the Dutch Obesity Intervention in Teenagers (NRG-DOiT): systematic development, implementation and evaluation of a school-based intervention aimed at the prevention of excessive weight gain in adolescents. BMC Public Health, 6:304. doi:10.1186/1471-2458-6-304

SLINGERLAND, M., \& BORGHOUTS, L. (2012). Direct and Indirect Influence of Physical Education-Based Interventions on Physical Activity: A Review. J Phys Act Health. , 8:6, pp. 866-878.

STRONG, W. B., MALINA, R. M., BLIMKIE, C. J., DANIELS, S. R., DISHMAN, R. K., GUTIN, B., HERGENROEDER, A. C., MUST, A., NIXON, P. A., PIVARNIK, J. M., ROWLAND, T., TROST, S. G., \& TRUDEAU, F. (2005). Evidence based physical activity for school-age youth. Journal of Pediatrics, 146:6, pp. 732-737.

TAYMOORI, P., NIKNAMI, S., BERRY, T., LUBANS, D., GHOFRANIPOUR, F., \& KAZEMNEJAD, A. (2008). A school-based randomized controlled trial to improve physical activity among Iranian high school girls. International Journal of Behavioral Physical Activity, 5. doi: 10.1186/1479-5868-5-18 
TELAMA, R. (2009). Tracking of Physical Activity from Childhood to Adulthood: A Review. Obesity Facts, 2:3, pp. 187-195.

TIMPERIO, A., SALMON, J., \& BALL, K. (2004). Evidence-based strategies to promote physical activity among children, adolescents and young adults: review and update. Journal of Science and Medicine in Sport, 7:1 Suppl, pp. 20-29.

TSORBATZOUDIS, H. (2005). Evaluation of a school-based intervention programme to promote physical activity: An application of the theory of planned behavior. Perceptual and Motor Skills, 101:3, pp. 787-802

VAN SLUIJS, E. M. F., MCMINN, A. M., \& GRIFFIN, S. J. (2008). Effectiveness of interventions to promote physical activity in children and adolescents: systematic review of controlled trials. British Journal of Sports Medicine, 42:8, pp. 653-657.

WALLHEAD, T. L., \& BUCKWORTH, J. (2004). The role of physical education in the promotion of youth physical activity. Quest, $56 \mathrm{pp} .285-301$.

WARD, D., SAUNDERS, R., FELTON, G., WILLIAMS, E., EPPING, J. N., \& PATE, R. R. (2006). Implementation of a school environment intervention to increase physical activity in high school girls. Health Education Research, 21:6, pp. 896-910.

WEBBER, L. S., CATELLIER, D. J., LYTLE, L. A., MURRAY, D. M., PRATT, C. A., YOUNG, D. R., ELDER, J. P., LOHMAN, T. G., STEVENS, J., JOBE, J. B., \& PATE, R. R. (2008). Promoting physical activity in middle school girls - Trial of activity for adolescent girls. American Journal of Preventive Medicine, 34:3, pp. 173-184. doi: 10.1016/j.amepre.2007.11.018

WECHSLER, H., DEVEREAUX, R. S., DAVIS, M., \& COLLINS, J. (2000). Using the school environment to promote physical activity and healthy eating. Preventive Medicine, 31:2, pp. S121-S137.

WHO. (2010). Global recommendations on physical activity for health. Geneva: WHO. Disponible en: http://whqlibdoc.who.int/publications/2010/9789241599979_eng.pdf. Fecha consulta: Dic 2010.

YOUNG, D. R., PHILLIPS, J. A., YU, T., \& HAYTHORNTHWAITE, J. A. (2006). Effects of a life skills intervention for increasing physical activity in adolescent girls. Archives of Pediatrics \& Adolescent Medicine, 160:12, pp. 1255-1261.

\section{Correspondencia con la autora}

Maria José CAMACHO-MIÑANO

Dpto. de Expresión Musical y Corporal

Facultad de Educación. Universidad Complutense de Madrid

C/ Rector Royo Villanova, s/n

E-28040 Madrid

Teléfono: 913946327

Correo-e: mjcamacho@edu.ucm.es 